\title{
Gremios y diversidad de hormigas (Hymenoptera: Formicidae) en tres usos del suelo de un paisaje cafetero del Cauca-Colombia.
}

\author{
Anderson Arenas-Clavijo ${ }^{1}$ \& Inge Armbrecht ${ }^{1}$ \\ 1. Departamento de Biología, Facultad de Ciencias Naturales y Exactas, Universidad del Valle, Calle 13 100-00 Edf. 320 \\ Cali-Colombia; anderson.arenas@correounivalle.edu.co, inge.armbrecht@correounivalle.edu.co
}

Recibido 17-IX-2017. Corregido 23-X-2017. Aceptado 22-XI-2017.

\begin{abstract}
Guilds and diversity of ants in three land uses from a coffee landscape at Cauca -Colombia. In the last 50 years, Colombian coffee production has undergone a great transformation from traditional plantations grown below the forest's shade, to vast extensions of coffee plantations consisting of free exposition to sun. These systems reduce native biodiversity and its associated ecosystem services. Simultaneously, shaded coffee plantations operate as potential shelter of such biodiversity, favoring its dispersal in the landscape, because they offer high-quality habitat for the movement of wild organisms through natural vegetation relicts. Therefore, it is important to document its biological value. In Colombia, Cauca department is the fourth coffee producer and although its cultures were mainly based on the model of using trees' shade on coffee shrubs, in last 10 years, sun coffee plantations have doubled their extension in this department and have almost equated the shaded coffee extension. Thus, it is necessary to document if these land use changes at landscape scale may derivate in consequences for the wild biota dwelling these productive systems. Given the above considerations, and in order to test for the biological importance of coffee plantations, the present study compares the effect of three land uses (sun coffee, shaded coffee and natural vegetation patches; eight of each one) in terms of ants' species richness and the abundance of their trophic guilds at Caldono municipality, between August 2015 and January 2016. Captures were carried out with pitfall traps, mini-Winkler sacs and direct capture. We found that ants' richness was higher in natural vegetation patches, followed by shaded coffee plantations and sun coffee plantations, in these last ones, the omnivore-generalist ants were more abundant, and ground-foraging arboreal ants were less abundant. We found that shaded coffee plantations, despite belonging to a transformed land use, have greater similarity with some natural vegetation patches. Using only ants from the lowest stratum (soil and understory), our findings confirm that shaded coffee plantations maintain an important part of the ant diversity of the locality, while sun coffee plantations offer less habitat quality to it. On the other hand, although natural vegetation patches are so small and degraded, their conservation represent a valuable heritage for the protection of local ants' fauna, harboring a large number of exclusive species. Rev. Biol. Trop. 66(1): 48-57. Epub 2018 March 01.
\end{abstract}

Key words: Coffee agroecosystems; predatory ants; tropical Andes; ant biodiversity; canopy remotion.

El café (Coffea arabica L. Rubiaceae) es una especie vegetal de gran importancia comercial en Colombia, cuya producción ubica este país como el tercero a nivel mundial, después de Brasil y Vietnam (ICO, 2017). Con el paso de los años, las variedades de los cafetos sembrados en Colombia -que en condiciones naturales de su sitio originario en África, crecen en el estrato bajo de bosques-, fueron progresivamente reemplazadas por variedades desarrolladas para resistir el pleno sol, sembradas a cielo abierto con arbustos bajos, en densidades de hasta 10000 por hectárea, y ocasionalmente acompañadas por una o dos especies vegetales, también productivas (Borrero, 1986; Armbrecht, 2016). Esta forma de producción proviene de una concepción de agricultura industrial, que depende de insumos químicos -fungicidas, herbicidas e insecticidas- para el óptimo desarrollo de los cultivos. El uso indiscriminado de este tipo de insumos y la exigencia de homogeneidad provoca una disminución de la calidad 
y cantidad de servicios ecológicos prestados por la diversidad biológica asociada a los agroecosistemas (Foley et al., 2011).

La estrategia de producción industrial ha desplazado a la tradicional bajo el argumento que el rendimiento en cafetales de sol es mayor que en cafetales de sombra (Perfecto, Rice, Greenberg, \& van der Voort, 1996; Rappole, King, \& Vega Rivera, 2003), esto ha causado que la diversidad biológica asociada sea menor en los cafetales de sol que en otras coberturas vegetales cercanas que incorporan árboles y mayor diversidad vegetal (Goehring, Daily, \& Şekerçioğlu, 2002). La estrategia tradicional para producir café consiste en la siembra de los cafetos a la sombra de varias especies arbóreas, en este caso, los arbustos son de mayor porte y se siembran en menor densidad, entre 5000 y 8000 por hectárea. Esto genera condiciones ambientales que favorecen la diversidad biológica asociada (Vergara \& Badano, 2009), pues los árboles acompañantes y su hojarasca amplían la variedad de microhábitats (Lavelle et al., 2006), incluyendo espacios para especies de estratos más altos, que ocasionalmente forrajean en el suelo (Ramírez, Herrera, \& Armbrecht, 2010).

En Colombia, más del $50 \%$ del área sembrada en café corresponde a cuatro departamentos: Huila, Antioquia, Tolima y Cauca, todos ellos ubicados en los Andes tropicales, una zona especialmente biodiversa (Herzog et al., 2011). En los tres primeros, la producción se basa en cafetales de sol, que alcanzan cerca del $90 \%$ del área cultivada en Huila, poco más del $70 \%$ en Antioquia y casi el $80 \%$ en Tolima. En Cauca, sin embargo, la extensión en cafetales de sombra (52820 ha) constituye todavía alrededor del $60 \%$ del área cultivada (FNC, 2017). Pese a esto, si se compara el crecimiento de las áreas cultivadas en función de su exposición al sol, la expansión de los cafetales de sol ha sido más del doble en los últimos 10 años.

Una forma de reconocer los efectos de la producción agrícola sobre la biodiversidad es contar las especies y compararlas entre usos del suelo. Los insectos son particularmente útiles para reconocer dichos efectos, porque son pequeños, numerosos y diversos (Samways, 2007). Las hormigas ocupan una importante porción de la diversidad de insectos, se reconocen 13258 especies válidas (Bolton, 2016), aunque su riqueza se estima en 25000 especies (Ward, 2009).

Las hormigas son consideradas importantes indicadores ecológicos (Andersen et al., 2004), de igual forma, exhiben una amplia gama de gremios tróficos y funcionales que operan en casi todos los estratos de los agroecosistemas (incluido el café), ofreciendo servicios ecosistémicos, como el control potenciales plagas, polinización o la introducción de materia orgánica en el suelo (Silvestre, Brandão, \& Rosa da Silva, 2003; Dias et al., 2013). Dado lo anterior, el objetivo fue identificar el efecto de los cafetales de sol y de sombrío sobre la riqueza y abundancia de hormigas y sus gremios tróficos, en un contexto climático de sequía extrema (Oscilación del Sur-El Niño 2014-2016).

\section{MATERIALES Y MÉTODOS}

Zona de estudio: La investigación se realizó en el suroccidente de Colombia, departamento Cauca, municipio Caldono, veredas El Rosal y El Cabuyal (2॰49'44" - 2'51'32"N

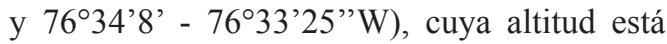
entre 1336 y $1538 \mathrm{msnm}$. La zona consistió en un mosaico de cultivos, con predominio de los cafetales de sombra sensu Moguel y Toledo (1999), especialmente los estados más intensificados. Se seleccionaron ocho cafetales de sombra con vegetación acompañante dominada por árboles adultos de Inga spectabilis (Vahl) e Inga edulis Mart. y de extensión variable (0.5-6 ha.), ocho cafetales de sol, en algunos casos acompañados por Musa x paradisiaca L., Manihot esculenta Crantz o Citrus x sinensis Osbeck, y en ninguno de éstos cultivos la diversidad vegetal acompañante fue mayor a tres especies (0.5-8 ha.). Así mismo, se seleccionaron ocho parches de vegetación natural como controles positivos, que consistieron en áreas arborizadas de tamaño no mayor a 2 ha., los mismos tuvieron un elevado 
grado de perturbación, pues la comunidad local extrae madera de éstos y permite el paso de ganado al interior de los mismos.

Diseño del muestreo: En cada repetición espacial (en adelante, parcela) se instalaron cinco trampas de caída y se realizaron cinco extracciones de $1 \mathrm{~m}^{2}$ de hojarasca para su cernido y separación en sacos mini-Winkler. Las trampas de caída consistieron de vasos de $266 \mathrm{~mL}$ llenos a $2 / 3$ de su capacidad con una solución 2:1 de agua y etanol al $96 \%$, que operaron por 48 horas, los sacos mini-Winkler operaron igual intensidad temporal. En adición, en cada parcela se realizó la búsqueda directa de especímenes en los árboles hasta la altura de $1.70 \mathrm{~m}$, y en otros espacios disponibles para la nidificación (bajo rocas, troncos caídos, ramitas, etc.), esta tuvo una intensidad de $30 \mathrm{~min} /$ investigador, para un total de $1 \mathrm{~h}$ de muestreo en cada parcela. Cada parcela se muestreó en dos ocasiones, una en agosto de 2015 y otra entre diciembre de 2015 y enero de 2016, durante este período de muestreo, la localidad estuvo influenciada por la Oscilación Sur- El Niño (ENSO), un período climático que causa extrema sequía en esta zona de América. Los especímenes recolectados se transportaron al Laboratorio de Biología y Ecología de Hormigas de la Universidad del Valle para su identificación y posterior depósito en el Museo de Entomología de la Universidad del Valle (Cali, Colombia).

Comparación de la riqueza de especies: Se construyeron curvas de acumulación de especies para cada uso del suelo, utilizando rarefacción basada en muestras, un método de interpolación que permite estimar la riqueza de especies esperada, con valores confiables de desviación estándar y que a su vez, permite establecer comparaciones entre distintos usos del suelo (Colwell, Mao, \& Chang, 2004). Para el caso de las hormigas, las curvas de rarefacción se calcularon con base en la incidencia de especies (número de registros), esto es, el número de veces que se capturó una especie en una parcela cualquiera, independiente de la cantidad de individuos (Gotelli, Ellison, Dunn, \& Sanders, 2011), las curvas y sus desviaciones estándar se calcularon con el programa EstimateS v8.2 (Colwell, 2013).

Efecto del uso del suelo sobre los gremios tróficos de hormigas: Las especies de hormigas se categorizaron en gremios tróficos de acuerdo con la literatura disponible (Delabie, Agosti, \& do Nascimento, 2000; Silvestre et al., 2003) (Cuadro 1), con esta información se construyeron modelos lineales generalizados mixtos de distribución Poisson, para determinar el efecto de cada uso del suelo sobre la abundancia de hormigas de cada gremio, este procedimiento se realizó con el paquete multcomp (Hothorn et al., 2016) de R (R Development Core Team, 2017).

Análisis de agrupamiento: La similitud entre usos del suelo en función de las especies registradas se calculó con un análisis de agrupamiento con el método de Bray-Curtis, usando el programa PAST (Hammer, Harper, \& Ryan, 2001). Finalmente se realizó un análisis SIMPER para identificar cuáles especies fueron las que más aportaron a la diferenciación entre los usos del suelo.

\section{RESULTADOS}

Comparación de la riqueza de especies: Se recolectaron 28726 individuos en 2494 registros, agrupadas en 113 especies de 43 géneros. La especie con mayor número de registros fue Wasmannia auropunctata (Roger, 1863) (285), seguida de Linepithema neotropicum Wild, 2007 (245). En general, las hormigas más abundantes fueron las omnívoras del suelo y hojarasca, con 1461 registros de 36 especies. Este gremio fue seguido de las depredadoras poneromorfas generalistas de suelo y hojarasca, con 312 registros de siete especies (ver anexo digital). La mayor cantidad de especies se encontró en parches de vegetación natural (86), seguidos de los cafetales de sombra (68) y los cafetales de sol (59) (Fig. 1), estas 
CUADRO 1

Listado de gremios tróficos de hormigas considerados en el presente estudio

TABLE 1

List of ants' trophic guilds considered in the present study

\begin{tabular}{|c|c|c|}
\hline Código & Descripción del gremio & Especies \\
\hline A & Omnívoras de suelo y hojarasca. & $\begin{array}{l}\text { Pheidole spp., Solenopsis spp., Wasmannia spp., Linepithema spp., } \\
\text { Cardiocondyla spp., Nesomyrmex asper, Temnothorax subditivus }\end{array}$ \\
\hline $\mathrm{B}$ & $\begin{array}{l}\text { Depredadoras poneromorfas } \\
\text { generalistas de suelo y hojarasca }\end{array}$ & $\begin{array}{l}\text { Odontomachus chelifer, Ectatomma ruidum, Neoponera verenae, } \\
\text { Neoponera aenescens, Pachycondyla impressa, Gnamptogenys } \\
\text { striatula }\end{array}$ \\
\hline $\mathrm{C}$ & Oportunistas de suelo y vegetación & $\begin{array}{l}\text { Camponotus spp., Nylanderia spp., Brachymyrmex spp., } \\
\text { Dorymyrmex brunneus }\end{array}$ \\
\hline $\mathrm{D}$ & $\begin{array}{l}\text { Cultivadoras de hongo a partir } \\
\text { de hojas cortadas }\end{array}$ & Atta cephalotes \\
\hline $\mathrm{E}$ & $\begin{array}{l}\text { Cultivadoras de hongo a partir } \\
\text { de detritus de artrópodos }\end{array}$ & Cyphomyrmex spp., Mycocepurus smithii, Apterostigma pilosum \\
\hline $\mathrm{F}$ & $\begin{array}{l}\text { Myrmicinas crípticas depredadoras } \\
\text { especializadas }\end{array}$ & Strumigenys spp., Acanthognathus ocellatus, Octostruma balzani \\
\hline G & $\begin{array}{l}\text { Depredadoras poneromorfas } \\
\text { crípticas }\end{array}$ & $\begin{array}{l}\text { Hypoponera spp., Heteroponera inca, Rasopone ferruginea, } \\
\text { Neoponera crenata, Discothyrea denticulata }\end{array}$ \\
\hline $\mathrm{H}$ & Especialistas crípticas del suelo & Acropyga spp., Tranopelta gilva \\
\hline I & Especies nómadas & Eciton burchellii foreli, Labidus spp., Neivamyrmex adnepos \\
\hline $\mathrm{J}$ & $\begin{array}{l}\text { Omnívoras arbóreas que } \\
\text { ocasionalmente forrajean } \\
\text { en estratos más bajos }\end{array}$ & $\begin{array}{l}\text { Crematogaster spp., Azteca sp., Myrmelachista zeledoni, } \\
\text { Procryptocerus spp., Cephalotes minutus }\end{array}$ \\
\hline $\mathrm{K}$ & Pseudomyrmecinas & Pseudomyrmex spp. \\
\hline
\end{tabular}

observaciones fueron confirmadas con rarefacción de especies basada en muestras (Fig. 2).

Efecto del uso del suelo sobre los gremios tróficos de hormigas: Si bien las hormigas omnívoras de suelo y hojarasca (A) predominaron en los tres usos del suelo, el análisis con Modelos Lineales Generalizados Mixtos mostró que este gremio fue más abundante en cafetales de sol, comparadas con los otros dos usos del suelo. Por su parte, las hormigas cortadoras de hojas (D) fueron más abundantes en parches de vegetación natural, mientras que las hormigas cultivadoras de hongos a partir de detritus de artrópodos (E) fueron más escasas en parches de vegetación natural, lo mismo que las mirmicinas crípticas depredadoras especializadas (F). Por otro lado, los cafetales de sol tuvieron las abundancias más bajas de hormigas especialistas crípticas del suelo $(\mathrm{H})$ y de omnívoras arbóreas (J) (Cuadro 2).

Análisis de agrupamiento: Los cafetales de sol formaron casi todos, un grupo consistente y bien diferenciado de los demás usos del suelo. Por su parte, seis de los parches de vegetación natural se agruparon con una similitud mínima cercana al $45 \%$. Los cafetales de sombra por su parte, no formaron alguna unidad consistente, sino que se mezclaron con los otros usos del suelo, especialmente con parches de vegetación natural (Fig. 3). El análisis SIMPER mostró que $W$. auropunctata, L. neotropicum y Solenopsis geminata (Fabricius, 1804) fueron las especies que más contribuyeron a las diferencias apreciadas en el 


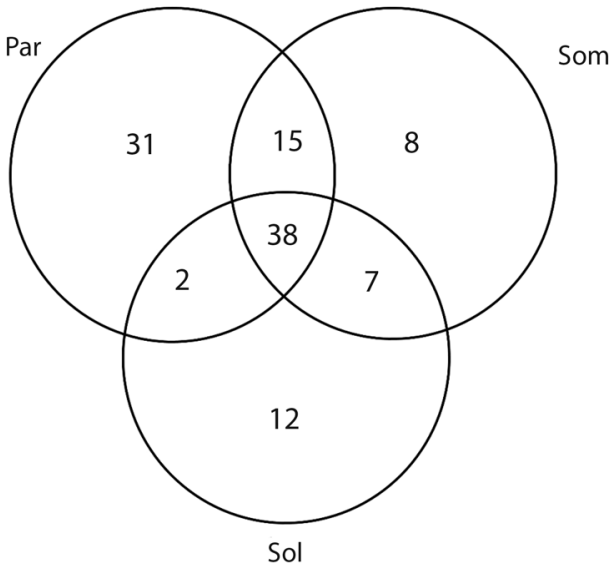

Fig. 1. Distribución de la riqueza de especies de hormigas por uso del suelo. Par: parche de vegetación natural, Som: cafetal de sombra, Sol: cafetal de sol.

Fig. 1. Ants species richness distribution by land use. Par: patch of natural vegetation, Som: shade coffee, Sun: sun coffee. análisis de agrupamiento de Bray-Curtis (cerca del $20 \%$ entre las tres), mientras que la mitad de las diferencias apreciadas recayó sobre un conjunto de 14 especies (ocho omnívoras del suelo y hojarasca, tres depredadoras poneromorfas del suelo y hojarasca, dos cultivadoras de hongo a partir de detritus y una myrmicina críptica depredadora especializada).

\section{DISCUSIÓN}

La riqueza total de especies de hormigas del presente estudio (113) fue mayor que la registrada por Urrutia-Escobar y Armbrecht (2013), Colombia en la misma localidad (82), quienes hicieron sus capturas únicamente con cebos de atún y trampas de caída. $\mathrm{La}$ mayor riqueza registrada en nuestro estudio podría deberse al uso de la captura manual

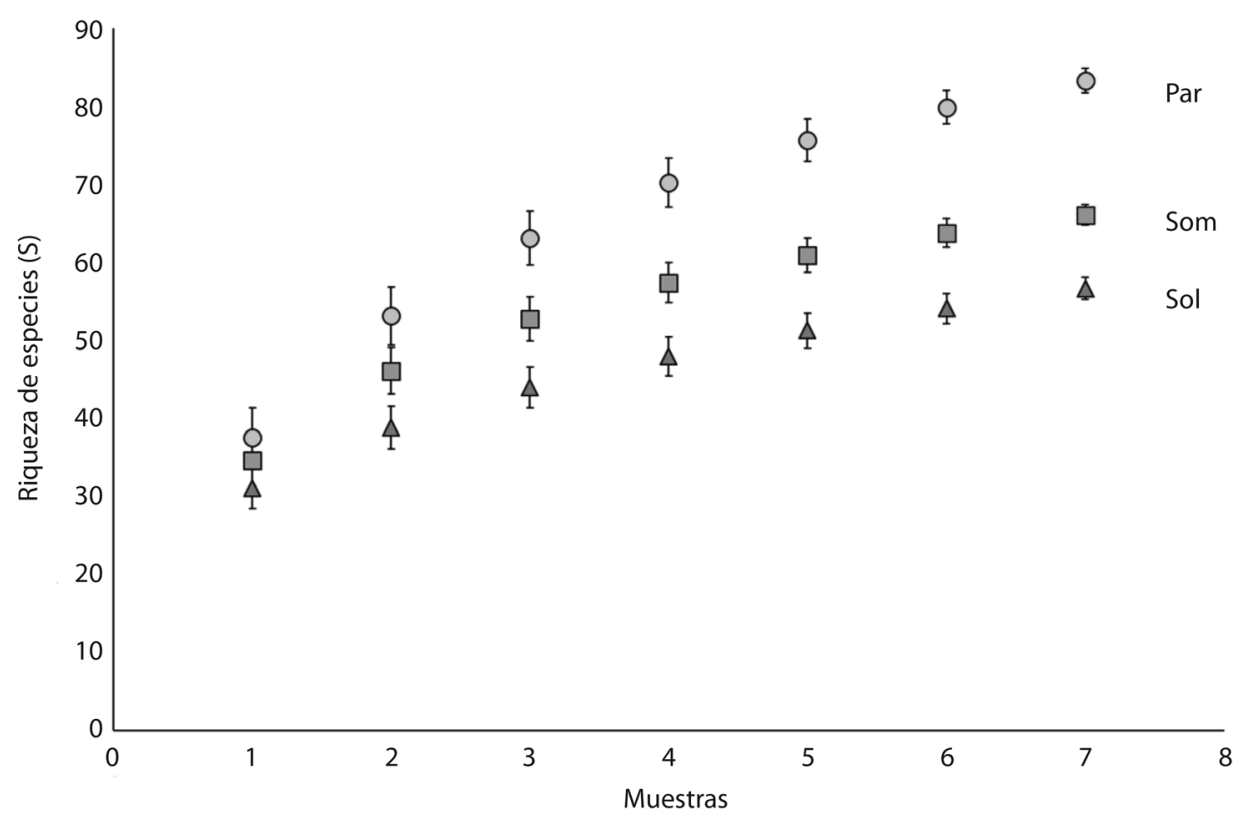

Fig. 2. Acumulación de especies de hormigas de acuerdo con la rarefacción basada en muestras. Las barras de error representan la desviación estándar (basada en la simulación de 100 remuestreos con remplazamiento). Par: parche de vegetación natural, Som: cafetal de sombra, Sol: Cafetal de sol.

Fig. 2. Ants species accumulation according to the rarefaction based on samples. The error bars represent the standard deviation (based on the simulation of 100 resampling with replacement). Par: natural vegetation patch, Som: shade coffee, Sun: Sun coffee. 
CUADRO 2

Abundancia promedio de hormigas \pm desviación estándar, de acuerdo con sus gremios tróficos. Los gremios corresponden a los descritos en el cuadro 1. Dev: devianza, P(chi): significancia de las diferencias halladas con el modelo lineal generalizado de distribución Poisson con 2 grados de libertad. Sol: cafetal de sol, Som: cafetal de sombra, Par: parche de vegetación natural

TABLE 2

Average abundance of ants \pm standard deviation, according to trophic guilds. Trophic guilds belong to these described in Table 1. Dev: deviance, P(chi): significance of differences found with general lineal model of Poisson distribution with 2 degrees of freedom. Sol: sun coffee, Som: Shaded coffee, Par: natural vegetation patch

\begin{tabular}{cccccc} 
Gremio & Dev & P(Chi) & Sol & Som & Par \\
A & 25.904 & $<0.001$ & $72.5 \pm 15.96 \mathrm{a}$ & $55 \pm 10.23 \mathrm{~b}$ & $55.125 \pm 17.62 \mathrm{~b}$ \\
B & 4.0846 & 0.1297 & $13.5 \pm 4.54$ & $14.5 \pm 8.57$ & $11 \pm 8.04$ \\
C & 1.8401 & 0.3985 & $9.75 \pm 7.92$ & $11 \pm 6.05$ & $8.875 \pm 3.04$ \\
D & 12.662 & $<0.01$ & $0.125 \pm 0.35 \mathrm{a}$ & $0.375 \pm 0.74 \mathrm{ab}$ & $1.5 \pm 1.51 \mathrm{~b}$ \\
E & 26.899 & $<0.001$ & $10.125 \pm 5.08 \mathrm{a}$ & $6.875 \pm 3.87 \mathrm{a}$ & $3.5 \pm 2.51 \mathrm{~b}$ \\
F & 16.294 & $<0.001$ & $6.75 \pm 4.71 \mathrm{a}$ & $4.875 \pm 3.04 \mathrm{a}$ & $2.5 \pm 2.33 \mathrm{~b}$ \\
G & 1.9404 & 0.379 & $2.625 \pm 3.66$ & $1.625 \pm 1.85$ & $2 \pm 2.77$ \\
H & 18.225 & $<0.001$ & $0.125 \pm 0.35 \mathrm{a}$ & $2.125 \pm 1.25 \mathrm{~b}$ & $1.625 \pm 1.51 \mathrm{~b}$ \\
I & 0.14665 & 0.9293 & $0.625 \pm 1.06$ & $0.625 \pm 0.92$ & $0.5 \pm 0.93$ \\
J & 27.299 & $<0.001$ & $0.625 \pm 1.19 \mathrm{a}$ & $1.375 \pm 0.92 \mathrm{a}$ & $4.25 \pm 2.05 \mathrm{~b}$ \\
K & 2.6618 & 0.2642 & $1.875 \pm 1.55$ & $2.5 \pm 2.07$ & $1.375 \pm 1.85$ \\
\hline
\end{tabular}

Las distintas letras al lado de los promedios indican diferencias estadísticamente significativas (Tukey, $\mathrm{P}<0.05$ ).

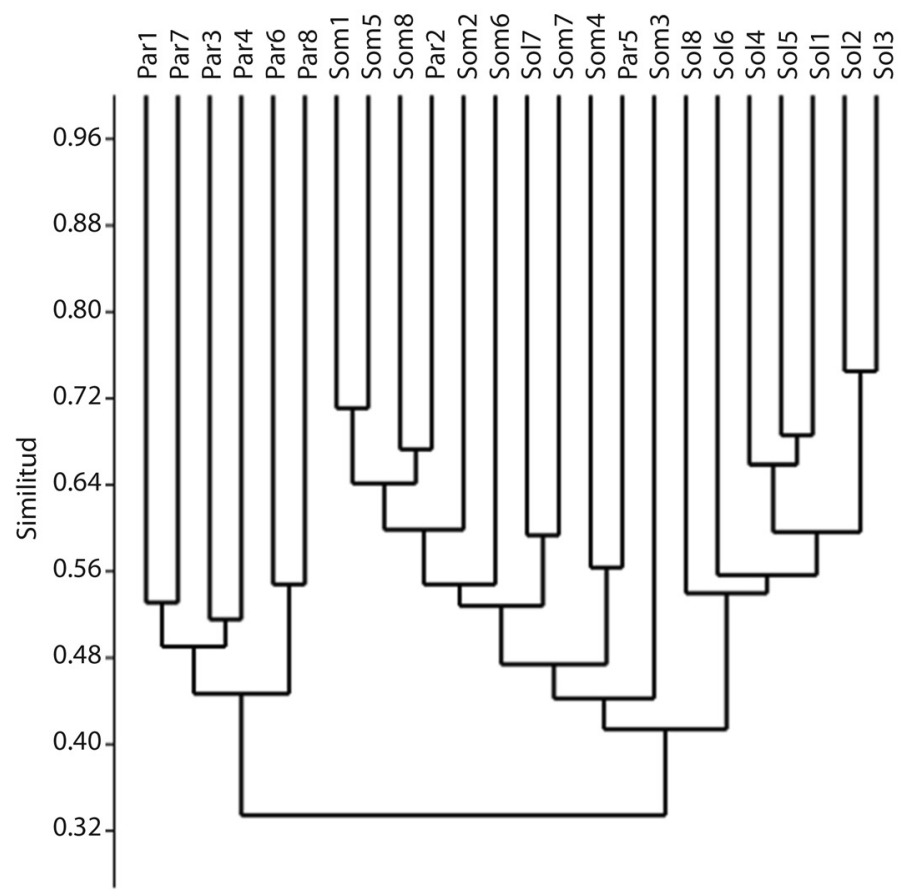

Fig. 3. Agrupamiento de los usos de suelo en función de las especies de hormigas del suelo y vegetación acompañante, según el método de Bray-Curtis.

Fig. 3. Land uses grouping by species of soil ants and accompanying vegetation, according to the Bray-Curtis method. 
de especímenes, que aporta algunas especies de hormigas que usualmente no se hallan con métodos de captura pasivos del estrato epigeo. La riqueza de hormigas registrada en este estudio fue ligeramente menor a lo reportado por Rivera y Armbrecht (2005), quienes registraron 115 especies capturadas con cernido de hojarasca y captura manual, de similares usos del suelo en Apía-Risaralda (la mayoría de las generalistas en cafetales de sol), y un poco mayor a lo encontrado por Zabala, Arango, y Chacón de Ulloa (2013): 96 especies de hojarasca, recolectadas con sacos Winkler en bosque continuo, parches de bosque y cafetales de sol, en La Celia-Risaralda. En este último, contrario a lo encontrado para el presente estudio, las hormigas omnívoras y depredadoras generalistas tuvieron mayor riqueza y abundancia de especies en coberturas boscosas.

Para el presente estudio, la riqueza específica de hormigas fue mayor en los parches de bosque que en los demás usos del suelo. Del total de especies registradas en éstos (86 especies), el $44 \%$ correspondió a especies halladas en todos los usos del suelo, y el $36 \%$ (31 especies) fueron exclusivas de parches. Comparativamente, las especies que compartidas entre todos los usos del suelo (38) correspondieron al 64 y al $56 \%$ de las especies registradas en cafetales de sol y de sombra respectivamente, evidenciando que parte de la fauna silvestre efectivamente pasa entre los cafetales y la vegetación natural. Es más, si se comparan las especies compartidas de forma exclusiva entre parches de vegetación y cafetales de sombra (15), con las de parches y cafetales de sol (2), se puede comprender el efecto amortiguador que los cafetales de sombra tienen sobre la diversidad de hormigas, incluso en condiciones climáticas adversas, donde los cafetales de sol se podrían ver más afectados.

La especie más abundante fue $W$. auropunctata, quien junto a $L$. neotropicum constituyeron poco más del $20 \%$ de los registros de este estudio, ambas especies son consideradas generalistas y a menudo se les asocia con problemas, entre otros, $W$. auropunctata se ha reportado como indicadora de baja diversidad
(Armbrecht \& Ulloa-Chacón, 2003), y esta especie junto a $L$. neotropicum, puede tener interacciones mutualistas con hemípteros de la familia Pseudococcidae, problemáticos para varios sistemas agrícolas (Mera Velasco, Gallego Ropero, \& Armbrecht, 2010). Por otro lado, entre las 31 especies consideradas raras dentro de este estudio (especies con dos o menos registros), 17 fueron encontradas exclusivamente en parches de vegetación natural. Esto sugiere que los parches de vegetación de la localidad estudiada, pese a ser pequeños, intervenidos y aislados, son de gran valor para la conservación biológica de hormigas.

El método de rarefacción basado en muestras mostró que, con solo ocho parcelas, las diferencias en la riqueza específica son estadísticamente significativas, con una menor riqueza de especies de cafetales de sol, respecto a los cafetales de sombra y parches de vegetación natural, tendencia observada a medida que el número de muestras aumentó. Este patrón concuerda con las bajas riquezas registradas en áreas abiertas, cuando se comparan con usos del suelo más arborizados (Cuautle, Vergara, \& Badano, 2016).

Las hormigas generalistas fueron más abundantes en los cafetales de sol. Este uso del suelo, a su vez, tuvo una baja cantidad de hormigas arbóreas y especialistas del suelo. Por su parte, otros grupos también considerados generalistas en un sentido amplio (especies oportunistas o depredadoras generalistas), tuvieron abundancias similares en los tres usos del suelo. Esto afirma la idea que las especies generalistas se benefician de los hábitats más degradados. Esta suma de fenómenos es comprensible si se considera el efecto de borde que, en paisajes fragmentados, promueve el ingreso de especies generalistas a parches de vegetación muy pequeños (Fahrig, 2003). Además, se encontró que la abundancia de hormigas cortadoras Atta cephalotes (Linnaeus, 1758) fue mayor en los parches de vegetación natural, situación que se explica porque esta especie presenta elevadas densidades de colonias en bordes de bosques, y su acción de herbivoría es 
mayor en los mismos (Urbas, Araújo, Leal, \& Wirth, 2007; Wirth et al., 2007).

La mayoría de los parches de vegetación natural formaron un grupo bien diferenciado, sin embargo, se debe destacar que las similitudes apreciadas entre los mismos fueron menores que las observadas en los cafetales de sol. Esto indica que hay especies de hormigas de parches de vegetación que no aparecen en otros, y que las especies de cafetales de sol son más homogéneas entre sí. Por otro lado, los cafetales de sombra formaron grupos entremezclados con parches de vegetación natural, a la vez que tuvieron mayor similitud con los cafetales de sol. Esto sugiere que los cafetales de sombra, operan como estados intermedios de perturbación entre áreas muy transformadas y áreas naturales. Finalmente, las especies generalistas fueron las que contribuyeron en mayor medida a las diferencias apreciadas en el análisis de agrupamiento, demostrando que este paisaje, más que presentar parches de vegetación muy conservados y con abundantes especies compartidas entre sí, está fuertemente dominado por las especies propias de áreas perturbadas que pueden ingresar a los usos del suelo más arborizados. Por lo anterior, sería de gran importancia considerar la restauración de estos pequeños parches de vegetación natural como una prioridad en la zona cafetera.

Nuestros hallazgos muestran que la instauración de sistemas abiertos, como los cafetales de sol, contribuyen menos a la conservación de la mirmecofauna de los ecosistemas naturales, no sólo por la eliminación de las especies vegetativas arbóreas y sus hormigas asociadas, sino también por los efectos secundarios que pueden tener las especies de hormigas propias de áreas abiertas en los pequeños parches de vegetación. A su vez, los cafetales de sol resultan en la simplificación la biodiversidad funcional asociada a los mismos, favoreciendo la abundante presencia de sólo un puñado de especies generalistas que domina en la localidad. Los cafetales de sombra por otro lado, pese a no albergar una alta cantidad de especies, ayudan a mitigar el efecto de la transformación del paisaje, al propiciar espacios para la presencia de algunas especies de parches de vegetación natural. Las especies exclusivas de parches de vegetación natural (junto con sus potenciales servicios ecosistémicos) se ven aglutinadas en sitios cada vez más degradados, y están condenadas a desaparecer del paisaje, de continuar presión antrópica en su interior y alrededores.

\section{AGRADECIMIENTOS}

El proyecto de convocatoria interna de la Universidad del Valle "Beneficios ecológicos de los cafetales de sombra en Colombia" CI 71000, el programa de Ciencias Básicas de Colciencias, proyecto $648-2013$ y la convocatoria 706 de Colciencias "Jóvenes Investigadores e Innovadores" año 2015 financiaron la presente investigación. Los autores agradecen los comentarios de los revisores anónimos, quienes aportaron sus conocimientos para mejorar la calidad de la presente contribución.

\section{RESUMEN}

En los últimos 50 años, la producción cafetera colombiana ha sufrido una gran transformación de los cultivos tradicionales crecidos a la sombra de los bosques, para derivarlos en vastas extensiones de cafetales de libre exposición al sol. Estos sistemas reducen la biodiversidad nativa y sus servicios ecosistémicos asociados. A su vez, los cafetales de sombrío operan como potenciales refugios de dicha biodiversidad, favoreciendo su dispersión en el paisaje dado que ofrecen hábitat de alta calidad para que los organismos silvestres se muevan a través de los relictos de vegetación natural, es por tanto importante documentar su valor biológico. En Colombia, el departamento del Cauca es el cuarto productor de café y aunque sus cosechas estaban principalmente basadas en el modelo de uso de sombra de árboles sobre cafetos, en los últimos 10 años los cafetales de sol han doblado su extensión en este departamento y casi han equiparado la extensión de los cafetales de sombra. Por lo tanto, es necesario documentar si estos cambios de uso de suelo a escala de paisaje pueden derivar en consecuencias para la biota silvestre que habita estos sistemas productivos. Dadas las anteriores consideraciones, y para probar la importancia biológica de las plantaciones de café, el presente estudio compara el efecto de tres usos del suelo (cafetales de sol, cafetales de sombra y parches de vegetación natural) en términos de la riqueza de especies de hormigas y la abundancia de sus gremios tróficos en 
el municipio de Caldono entre agosto de 2015 y enero de 2016. Las capturas fueron realizadas con trampas de caída, sacos mini-Winkler y colecta directa. Encontramos que la riqueza de hormigas fue mayor en parches de vegetación natural, seguidos de cafetales de sombra y cafetales de sol. En estos últimos, las hormigas omnívoras generalistas fueron más abundantes, y las hormigas arbóreas que forrajean en el suelo fueron menos abundantes. Por su parte, los cafetales de sombra, pese a pertenecer a un uso del suelo transformado, guardan similitud con algunos parches de vegetación natural. Usando sólo hormigas del estrato más bajo (suelo y sotobosque), nuestros hallazgos confirman que los cafetales de sombra mantienen una parte importante de la diversidad de hormigas de la localidad, mientras que los cafetales de sol ofrecen menor calidad de hábitat para la misma. Por otro lado, pese a que los parches de vegetación natural son tan pequeños y degradados, su conservación representa un valioso patrimonio para la protección de la fauna de hormigas local, albergando una gran cantidad de especies exclusivas.

Palabras clave: Conservación biológica en agroecosistemas; gremios tróficos; Andes tropicales

\section{REFERENCIAS}

Andersen, A., Hoffmann, B.D., Müller, W.J., \& Griffiths, A.D. (2004). Using ants as bioindicators in land management: simplifying assessment of ant community responses. Journal of Applied Ecology, 39, 8-17.

Armbrecht, I. (2016). Agroecología y Biodiversidad. Cali, Colombia: Programa Editorial Universidad del Valle.

Armbrecht, I., \& Ulloa-Chacón, P. (2003). The Little Fire ant Wasmannia auropunctata (Roger) (Hymenoptera: Formicidae) as a Diversity Indicator of Ants in Tropical Dry Forest Fragments of Colombia. Environmental Entomology, 32(3), 542-547.

Bolton, B. (2016). An online catalog of the ants of the world. Recuperado de http://antcat.org.

Borrero, J. I. (1986). La substitucion de cafetales de sombrío por caturrales y su efecto negativo sobre la fauna de vertebrados. Caldasia, 15(71-75), 725-732.

Colwell, R. (2013). EstimateS: Statistical estimation of species richness and shared species from samples. User's Guide and application. Recuperado de http:// purl.oclc.org/estimates

Colwell, R. K., Mao, C. X., \& Chang, J. (2004). Interpolating, extrapolating, and comparing incidencebased species accumulation curves. Ecology, 85(10), 2717-2727.

Cuautle, M., Vergara, C. H., \& Badano, E. I. (2016). Comparison of ant community diversity and functional group composition to land use change in a seasonally dry oak forest. Neotropical Entomology, 45(2), 170-179.

Delabie, J. H. C., Agosti, D., \& do Nascimento, I. C. (2000). Litter ant communities of the Brazilian Atlantic rain forest region. In D. Agosti, J. D. Majer, L. E. Alonso, \& T. R. Schultz (Eds.), Sampling ground dwelling ants: Case studies from the World's rain forests (pp. 1-17). Washington: Smithsonian Institution Press.

Dias, N. D. S., Zanetti, R., Santos, M. S., Peñaflor, M. F. G. V., Broglio, S. M. F., \& Delabie, J. H. C. (2013). The impact of coffee and pasture agriculture on predatory and omnivorous leaf-litter ants. Journal of Insect Science (Online), 13, 29. doi:10.1673/031.013.2901

Fahrig, L. (2003). Effects of habitat fragmentation on biodiversity. Annual Review of Ecology and Systematics, 34(May), 487-515.

Federación Nacional de Cafeteros de Colombia (FNC) (2017). Área cultivada según exposición solar por departamento - anual desde 2007. Recuperado de https://www.federaciondecafeteros.org/particulares/ es/quienes_somos/119_estadisticas_historicas/

Foley, J. A., Ramankutty, N., Brauman, K. A., Cassidy, E. S., Gerber, J. S., Johnston, M., ... Zaks, D. P. M. (2011). Solutions for a cultivated planet. Nature, 478, 337-342.

Goehring, D. M., Daily, G. C., \& Şekerçioğlu, Ç. H. (2002). Distribution of ground-dwelling arthropods in tropical countryside habitats. Journal of Insect Conservation, 6, 83-91.

Gotelli, N. J., Ellison, A. M., Dunn, R. R., \& Sanders, N. J. (2011). Counting ants (Hymenoptera: Formicidae): Biodiversity sampling and statistical analysis for myrmecologists. Myrmecological News, 15(May), 13-19.

Hammer, Ø., Harper, D. a. T., \& Ryan, P. D. (2001). Paleontological statistics software package for education and data analysis. Palaeontologia Electronica, 4(1), 9-18.

Herzog, S. K., Martínez, R., Jørgensen, P. M., \& Tiessen, H. (2011). Climate change and biodiversity in the tropical Andes. Inter-American Institute for Global Change Research (IAI) and Scientific Committee on Problems of the Environment (SCOPE).

Hothorn, T., Bretz, F., Westfall, P., Heiberger, R. M., Schuetzenmeister, A., \& Scheibe, S. (2016). Package “ multcomp "Version 1.4-6.

International Coffee Organization (ICO) (2017). Historical data on the global coffee trade, 1990-Present. Recuperado de http://www.ico.org/new_historical. asp?section $=$ Statistics 
Lavelle, P., Decaëns, T., Aubert, M., Barot, S., Blouin, M., Bureau, F., ... Rossi, J. P. (2006). Soil invertebrates and ecosystem services. European Journal of Soil Biology, 42(SUPPL. 1), S3-S15. doi:10.1016/j. ejsobi.2006.10.002

Mera Velasco, Y. A., Gallego Ropero, M. C., \& Armbrecht, I. (2010). Interacciones entre hormigas e insectos en follaje de cafetales de sol y sombra, Cauca-Colombia. Revista Colombiana de Entomología, 36(1), 116-126.

Moguel, P., \& Toledo, V. M. (1999). Biodiversity conservation in traditional coffee systems of Mexico. Conservation biology, 13(1), 11-21.

Perfecto, I., Rice, R., Greenberg, R., \& van der Voort, M. (1996). Shade coffee: A dissapearing refuge for biodiversity. BioScience, 46(8), 598-608.

R Development Core Team. (2017). R: A language and environment for statistical computing. Vienna, Austria: R Foundation for Statistical Computing.

Ramírez, M., Herrera, J., \& Armbrecht, I. (2010). ¿Bajan de los árboles las hormigas que depredan en potreros y cafetales Colombianos? Revista Colombiana de Entomologia, 36(1), 106-115.

Rappole, J. H., King, D. I., \& Vega Rivera, J. H. (2003). Coffee and conservation. Conservation Biology, 17(1), 334-336.

Rivera, L., \& Armbrecht, I. (2005). Diversidad de tres gremios de hormigas en cafetales de sombra, de sol y bosques de Risaralda. Revista Colombiana de Entomología, 31(1), 89-96.

Samways, M. J. (2007). Insect conservation: a synthetic management approach. Annual Review of Entomology, 52, 465-487.
Silvestre, R., Brandão, C. R. F., \& Rosa da Silva, R. (2003). Grupos funcionales de hormigas: el caso de los gremios del Cerrado. Introducción a las hormigas de la región Neotropical, 7, 113-148.

Urbas, P., Araújo, M., Leal, I., \& Wirth, R. (2007). Cutting more from cut forests: edge effects on foraging and herbivory of leaf-cutting ants in brazil. Biotropica, 39(4), 489-495.

Urrutia-Escobar, M. X., \& Armbrecht, I. (2013). Effect of two agroecological management strategies on ant (Hymenoptera: Formicidae) diversity on coffee plantations in southwestern Colombia. Environmental Entomology, 42(2), 194-203.

Vergara, C. H., \& Badano, E. I. (2009). Pollinator diversity increases fruit production in Mexican coffee plantations: The importance of rustic management systems. Agriculture, Ecosystems and Environment, 129, 117-123.

Ward, P. S. (2009). Taxonomy, Phylogenetics, and Evolution. In L. Lach, C. L. Parr, \& K. Abbott (Eds.), Ant Ecology (pp. 3-17). New York: Oxford University Press.

Wirth, R., Meyer, S. T., Almeida, W. R., Ara, M. V., Barbosa, V. S., Leal, I. R., ... Al, E. T. (2007). Increasing densities of leaf-cutting ants (Atta spp.) with proximity to the edge in a Brazilian Atlantic forest. Journal of Tropical Ecology, 23, 501-505.

Zabala, G. A., Arango, L. M., \& Chacón de Ulloa, P. (2013). Diversidad de hormigas (Hymenoptera: Formicidae) en un paisaje cafetero de Risaralda, Colombia. Revista Colombiana de Entomología, 39(1), 141-149. 